\title{
INTEGRAL and Swift/XRT observations of the source PKS 0208-512
}

\author{
S. Zhang ${ }^{1}$, W. Collmar ${ }^{2}$, D. F. Torres ${ }^{3}$, J.-M. Wang ${ }^{1,4}$, M. Lang ${ }^{2}$, and S.-N. Zhang ${ }^{1}$ \\ 1 Key Laboratory for Particle Astrophysics, Institute of High Energy Physics, Beijing 100049, PR China \\ e-mail: szhang@mail.ihep.ac.cn \\ 2 Max-Planck-Institut für Extraterrestrische Physik, PO Box 1603, 85740 Garching, Germany \\ 3 ICREA \& Institut de Ciències de l'Espai (IEEC-CSIC), Campus UAB, Facultat de Ciències, Torre C5-parell, 2a planta, \\ 08193 Barcelona, Spain \\ 4 Theoretical Physics Center for Science Facilities (TPCSF), CAS, PR China
}

Received 12 November 2009 / Accepted 9 February 2010

ABSTRACT

\begin{abstract}
Aims. The active galaxy PKS 0208-512, detected at lower energies by COMPTEL, has been claimed to be a MeV blazar from EGRET. We report on the most recent INTEGRAL observations of the blazar PKS 0208-512, which are supplemented by Swift ToO observations.

Methods. The high energy X-ray and $\gamma$-ray emission of PKS 0208-512 during August-December 2008 has been studied using 682 ks of INTEGRAL guest observer time and $\sim 56 \mathrm{ks}$ of Swift/XRT observations. These data were collected during the decay of a $\gamma$-ray flare observed by Fermi/LAT.

Results. At X-ray energies (0.2-10 keV) PKS 0208-512 is significantly detected by Swift/XRT, showing a power-law spectrum with a photon index of $\sim 1$.64. Its X-ray luminosity varied by roughly $30 \%$ during one month. At hard X-/soft $\gamma$-ray energies PKS $0208-512$ shows a marginally significant $(\sim 3.2 \sigma)$ emission in the $0.5-1 \mathrm{MeV}$ band when combining all INTEGRAL/SPI data. Non-detections at energies below and above this band by INTEGRAL/SPI may indicate intrinsic excess emission. If this possible excess is produced by the blazar, one possible explanation could be that its jet consists of an abundant electron-positron plasma, which may lead to the emission of an annihilation radiation feature. Assuming this scenario, we estimate physical parameters of the jet of PKS 0208-512.
\end{abstract}

Key words. X-rays: individuals: PKS 0208-512

\section{Introduction}

It has been suggested that relativistic jets of active galactic nuclei (AGN) may contain electron-positron pair plasmas (Begelman et al. 1984). The detection of the high energy $\gamma$-ray emission from a very compact region around the central supermassive black hole in M 87 (Aharonian et al. 2006; Acciari et al. 2009) suggested that electron-positron pair plasmas can be generated near black holes, thus setting up favorable conditions for launching electron-positron pair plasma jets. This is further supported by several AGN observations, like those of PKS 2155-304 and Mkn 501, which have been detected to process much shorter (3-5 min) variability (Aharonian et al. 2007; Albert et al. 2007). Reynolds et al. (1996) argued for the dominance of an electronpositron pair plasma in the jet of M 87 and thus in other AGNs, and e.g. Wardle et al. (1998), Hirotani et al. (2000), Lobanov \& Zensus (2001), Kino \& Takahara (2004), Dunn et al. (2006) studied different aspects of this eventuality. Other recent studies propose that extragalactic jets can also be dynamically dominated by cold protons (e.g., Celotti \& Ghisellini 2008). The determination of the jet content will certainly have important implications for understanding the mechanisms of jet launching, acceleration and collimation

The annihilation radiation in AGN jets may reveal itself in two different manners. Jets containing positrons hit a dense ambient medium, then the positrons can be thermalized and annihilate with the ambient electrons, thereby forming a narrow peak around $511 \mathrm{keV}$. Marscher et al. (2007) searched for such a narrow $(\leq 3 \mathrm{keV})$ emission feature in the spectrum of the radio galaxy 3C 120, in which the jet strongly interacts with interstellar clouds. They did not find a signal, and their upper limit did not constrain the positron-to-proton ratio in the jet. Alternatively, electrons and positrons may be already thermalized in the jets, and thus a broadened and blue-shifted annihilation component could show up on top of the spectral continuum in the $\mathrm{MeV}$ energy range (e.g., Böttcher \& Schlickeiser 1996; Skibo et al. 1997). During the Compton gamma ray observatory (CGRO) era, several AGNs were reported to show such broad bumps at $\mathrm{MeV}$ energies (e.g., Bloemen et al. 1995; Blom et al. 1995), which were called "MeV blazars" due to their excess emission at $\mathrm{MeV}$ energies. However, none of them passed subsequent data analysis checks (Blom et al. 1995; Williams et al. 2001; Stacy et al. 2003), and so these reports were discussed quite controversially. In fact, the first convincing signal of electron-positron annihilation radiation from AGN jets is yet to be found.

The source PKS 0208-512 is one such CGRO-detected $\gamma$-ray source claimed to be a MeV blazar. Two COMPTEL observations showed excess $\mathrm{MeV}$-emission (Blom et al. 1995) compared to the extrapolation of the EGRET spectrum, measured at energies above $100 \mathrm{MeV}$ (Bertsch et al. 1993; Skibo et al. 1997). The variability of the source above $100 \mathrm{MeV}$ was studied by von Montigny et al. (1995). They found a characteristic variability timescale of eight days, which suggested a $\gamma$-ray emission region on the order of several Schwarzschild radii for a black hole of $10^{10} M_{\odot}$. Further $\gamma$-ray observations and data analyses of this blazar led to the detection of persistent low-energy 
Table 1. INTEGRAL observations of PKS 0208-512.

\begin{tabular}{|c|c|c|c|c|c|}
\hline Rev. ID & Date & $\begin{array}{l}\text { MJD } \\
\text { (day) }\end{array}$ & SCWs & $\begin{array}{l}\text { Exposure } \\
\quad(\mathrm{ks})\end{array}$ & $\begin{array}{l}\text { Flux }(0.5-1 \mathrm{MeV}) \\
\left(\times 10^{-3} \mathrm{ph} \mathrm{cm}^{-2} \mathrm{~s}^{-1}\right)\end{array}$ \\
\hline 714 & 2008 Aug. $18-20$ & $54696.26-54698.44$ & 50 & 172 & \\
\hline 746 & 2008 Nov. $22-23$ & $54792.75-54793.75$ & 24 & 82 & $1.5 \pm 1.2$ \\
\hline 754 & 2008 Dec. $16-17$ & $54816.69-54817.77$ & 25 & 85 & $1.1 \pm 1.1$ \\
\hline 755 & 2008 Dec. $19-21$ & $54819.92-54820.28$ & 31 & 106 & $2.5 \pm 1.0$ \\
\hline 756 & 2008 Dec. $22-23$ & $54822.92-54823.74$ & 19 & 69 & $1.6 \pm 1.3$ \\
\hline 757 & 2008 Dec. $24-26$ & $54824.69-54826.78$ & 48 & 168 & $1.2 \pm 0.8$ \\
\hline $\begin{array}{l}\text { Exposure } \\
(\mathrm{ks})\end{array}$ & $\begin{array}{c}E \text {-band } \\
(\mathrm{MeV})\end{array}$ & $\begin{array}{c}\text { Flux } \\
\left(\times 10^{-4} \mathrm{ph} \mathrm{cm}^{-2} \mathrm{~s}^{-1}\right)\end{array}$ & \multicolumn{3}{|c|}{$\begin{array}{l}\text { INTEGRAL } \\
\text { (instrument) }\end{array}$} \\
\hline 510 & $\begin{array}{l}0.3-0.5 \\
0.5-1.0 \\
1.0-1.4 \\
1.4-8.0\end{array}$ & $\begin{array}{c}<6.4 \\
15.0 \pm 4.7 \\
<8.5 \\
<24.2\end{array}$ & \multicolumn{3}{|c|}{ SPI(imaging) } \\
\hline 510 & $\begin{array}{l}0.3-0.5 \\
0.5-1.0 \\
1.0-1.4\end{array}$ & $\begin{array}{c}<6.0 \\
14.6 \pm 4.5 \\
<9.3\end{array}$ & \multicolumn{3}{|c|}{ SPI(spectral) } \\
\hline 682 & $\begin{array}{c}0.02-0.04 \\
0.04-0.1 \\
0.1-0.3\end{array}$ & $\begin{array}{l}<0.7 \\
<0.7 \\
<1.6\end{array}$ & \multicolumn{3}{|c|}{ ISGRI } \\
\hline 682 & $\begin{array}{c}0.006-0.01 \\
0.01-0.02\end{array}$ & $\begin{array}{l}<17.0 \\
<17.6\end{array}$ & \multicolumn{3}{|c|}{ JEM-X } \\
\hline
\end{tabular}

Notes. The revolution ID, calendar date, time in MJD, number of science windows, exposure, energy band and flux are given. The errors are $1 \sigma$ and upper limits $2 \sigma$.

( $<3 \mathrm{MeV}) \mathrm{MeV}$ emission at a significance level of about $4 \sigma$ for the period 1991-1998 (Williams et al. 2001). A comparison to the contemporary EGRET spectrum ( $>100 \mathrm{MeV}$ ) (Hartman et al. 1999) indicated a MeV excess. The strength of this excess however is uncertain, because it varied with COMPTEL event selections (Williams et al. 2001). Furthermore, the statistical significance of this excess was challenged by analyses using all COMPTEL data (Stacy et al. 2003). So, even today there is still no unambiguous evidence for a $\mathrm{MeV}$ excess in the spectrum of PKS 0208-512, or any other blazar.

The satellite BeppoSAX observed PKS 0208-512 on 14th January 2001 and measured a spectral index of $1.64 \pm 0.10$ at soft $\mathrm{X}$-rays (Donato et al. 2005). The Chandra observation (Schwartz et al. 2006; Schwartz 2007) with an exposure of $~ 5$ ks showed a complex X-ray image, which can be resolved into at least four regions: the dominant core, two regions along the jet that may correspond to a hot spot and an extended lobe, and a possible fourth region outside the jet. By adopting a CMB (cosmic microwave background) model, where the X-rays are assumed to be produced via Comptonization of the cosmic microwave background photons by jet blob electrons, the jet Doppler factor was estimated as 5.7-7.3. The fit of the spectral energy distribution (SED), containing the 2008 Fermi data at energies above $200 \mathrm{MeV}$, resulted in a Lorentz factor of about 10 (Ghisellini et al. 2010).

We conducted INTEGRAL observations of PKS 0208-512 between August and December 2008 for a total of $\sim 682 \mathrm{ks}$. Since August 2008 the Fermi observatory (Atwood et al. 2009) surveys the sky at energies above $30 \mathrm{MeV}$ with its Large Area Telescope (LAT), thereby also monitoring PKS 0208-512 on a daily basis. The source showed a flare in 2008. The long-term monitoring in optical and near-IR bands revealed a significant brightening of PKS 0208-512, with an increase of 1.3 mag in the $B$-band and $1.4 \mathrm{mag}$ in the $R$-band between September 11 and 30, 2008 (Buxton et al. 2008). A contemporaneous increase in $\gamma$-rays was detected by Fermi (Tosti 2008). The publicly available $\gamma$-ray lightcurve ${ }^{1}$ shows a variable flux, up to about a factor of 6 on timescales of weeks. Our INTEGRAL observations were carried out during the decay of this flaring event. Supplementary to our INTEGRAL/SPI observations, we were granted two contemporaneous Swift ToO observations, providing the blazar state at softer X-ray energies. In this paper, we report on our findings.

\section{Observations and data analysis}

The ESA scientific mission INTEGRAL is dedicated to highresolution spectroscopy $(E / \Delta E \simeq 500$; SPI see Vedrenne et al. 2003) and imaging (angular resolution: 12' FWHM, point source location accuracy: $\simeq 1^{\prime}-3^{\prime}$; IBIS/ISGRI, see Ubertini et al. 2003; and Lebrun et al. 2003) of celestial sources in the energy range $15 \mathrm{keV}$ to $10 \mathrm{MeV}$. INTEGRAL also provides simultaneous monitoring at $\mathrm{X}$-rays $(3-35 \mathrm{keV}$, angular resolution: 3'; JEM-X, see Lund et al. 2003) and optical wavelengths (Johnson $V$-filter, $550 \mathrm{~nm}$; OMC, see Mas-Hesse et al. 2003). All INTEGRAL instruments, except OMC, work with coded masks. Observations of $682 \mathrm{ks}$ in total were carried out between August and December 2008 by applying the $5 \times 5$ dithering mode centered on PKS 0208-512. These data comprise 197 science windows (scw), each lasting typically for $3 \mathrm{ks}$. INTEGRAL/SPI was operational for about $510 \mathrm{ks}$ during these observations. Table 1 gives the details of the INTEGRAL observations. The data are analyzed with the INTEGRAL offline scientific analysis (OSA) version 7.0. For the spectral analysis of the SPI data we used SPIMODFIT version 3.0, which is now also available in OSA version 8.0.

The $\gamma$-ray burst explorer Swift was launched on November 20, 2004. It carries three co-aligned detectors (Gehrels et al. 2004), namely the Burst Alert Telescope (BAT, Barthelmy et al. 2005), the X-Ray Telescope (XRT, Burrows et al. 2005) and the Ultraviolet/Optical Telescope (UVOT,

\footnotetext{
1 At http://fermi.gsfc.nasa.gov/ssc/data/access/lat/ msl_lc
} 
Table 2. Swift/XRT observations of PKS 0208-512.

\begin{tabular}{|c|c|c|c|c|c|c|c|c|}
\hline $\begin{array}{c}\text { Obs. ID } \\
\text { (Swift/XRT) }\end{array}$ & Date & $\begin{array}{l}\text { MJD } \\
\text { (day) }\end{array}$ & $\begin{array}{c}\text { Offset } \\
\text { (arcsecond) }\end{array}$ & $\begin{array}{c}\text { Expo. } \\
\text { (ks) }\end{array}$ & Index & $\mathrm{N}$ & $\mathrm{L}$ & $\chi^{2} /$ d.o.f. \\
\hline 00035002001 & 2005 Apr. 23 & 53483.67 & 0.8 & 12.4 & & & & \\
\hline 00035002003 & 2005 May. 04 & 53495.00 & 1.1 & 2.1 & & & & \\
\hline 00035002004 & 2005 May. 12 & 53502.02 & 1.1 & 2.1 & & & & \\
\hline 00035002005 & 2005 May. 10 & 53500.01 & 1.3 & 4.2 & & & & \\
\hline combined & & & & 20.8 & $1.64 \pm 0.06$ & $4.4 \pm 0.2$ & 3.4 & $0.9 / 46$ \\
\hline 00035002014 & 2008 Oct. 23 & 54762.56 & 1.5 & 3.7 & & & & \\
\hline 00035002017 & 2008 Nov. 10 & 54780.65 & 2.3 & 3.8 & & & & \\
\hline 00035002021 & 2008 Nov. 25 & 54795.43 & 1.5 & 2.2 & & & & \\
\hline combined & & & & 9.7 & $1.63 \pm 0.11$ & $4.3 \pm 0.3$ & 3.4 & $1.28 / 19$ \\
\hline 00035002026 & 2008 Dec. 17 & 54817.31 & 4.1 & 4.2 & & & & \\
\hline 00035002027 & 2008 Dec. 20 & 54820.07 & 0.8 & 4.2 & & & & \\
\hline 00035002028 & 2008 Dec. 20 & 54820.73 & 0.4 & 4.4 & & & & \\
\hline 00035002029 & 2008 Dec. 23 & 54823.07 & 1.1 & 4.4 & & & & \\
\hline 00035002030 & 2008 Dec. 24 & 54824.81 & 0.2 & 4.2 & & & & \\
\hline 00035002031 & 2008 Dec. 26 & 54826.10 & 0.8 & 4.6 & & & & \\
\hline combined & & & & 26 & $1.64 \pm 0.07$ & $3.0 \pm 0.2$ & 2.3 & $1.2 / 38$ \\
\hline combined all & & & & 56.5 & $1.62 \pm 0.04$ & $3.9 \pm 0.1$ & 3.1 & $1.25 / 100$ \\
\hline combined 2008 & & & & 35.7 & $1.60 \pm 0.05$ & $3.3 \pm 0.1$ & 2.7 & $1.1 / 59$ \\
\hline
\end{tabular}

Notes. The observation ID, time in calendar date and MJD, the pointing offset angle, exposure, spectral index, normalization (in units of $10^{-4} \mathrm{ph} \mathrm{cm}^{-2} \mathrm{~s}^{-1} \mathrm{keV}^{-1}$ ), luminosity (in units of $10^{-12} \mathrm{erg} \mathrm{cm}^{-2} \mathrm{~s}^{-1}$, at $0.2-10 \mathrm{keV}$ ), and the reduced $\chi^{2}$ and degree of freedom (d.o.f.) of the power-law fits are given.

Roming et al. 2005). Between 2005 and 2008 Swift/XRT observed PKS 0208-512 13 times, exposing longer than $2 \mathrm{ks}$ (Table 2). This yielded a total exposure time of $\sim 56 \mathrm{ks}$. The 2008 observations were - by our ToO request - contemporaneous to the INTEGRAL observations. We selected all these observations for our studies, and analyzed the XRT data with Heasoft v. 6.2. For the spectral fitting we applied XSPEC v 12.3.1 and estimated the model parameters at the $90 \%$ confidence level.

\section{Results}

The source PKS 0208-512 was not detected by the INTEGRAL instruments IBIS/ISGRI and JEM-X, neither in individual scw nor in the sum of all data. However, the image of INTEGRAL/SPI, combining the whole data set ( 510 ks), reveals evidence for a detection at the $3.2 \sigma$ level in the $0.5-1 \mathrm{MeV}$ band (Fig. 1). A single source, exactly coincident in location with PKS 0208-512, is visible in the SPI map, which is otherwise rather empty and clean, at a flux level of $(1.50 \pm 0.47) \times$ $10^{-3} \mathrm{ph} \mathrm{cm}^{-2} \mathrm{~s}^{-1}$. The source is not visible with SPI in other energy ranges, i.e. below $0.5 \mathrm{MeV}$ and above $1 \mathrm{MeV}$. Maps in the $0.3-0.5 \mathrm{MeV}$ band and above $1.0 \mathrm{MeV}$ remain empty, i.e., yielding only upper flux limits. The SPI energy bands $0.3-0.5 \mathrm{MeV}$ and $0.5-1.0 \mathrm{MeV}$ adopted here are commonly used in SPI analyses. For example, Knödlseder et al. (2007) used 0.3-0.5 MeV and $0.514-1.0 \mathrm{MeV}$ for producing SPI all-sky maps; Bouchet et al. (2005, 2008) used 0.3-0.6 MeV and 0.6-1.0 MeV bands, and Petry et al. (2009) chose 0.278-0.502 MeV and $0.502-1.0 \mathrm{MeV}$ for imaging SPI sources. We selected our bands before the analysis, based on these typical SPI choices ${ }^{2}$.

\footnotetext{
2 Above $1.0 \mathrm{MeV}$ we took the high-energy band limit as $1.4 \mathrm{MeV}$ because the sensitivity above $\sim 1.4 \mathrm{MeV}$ worsens (see the SPI user manual, Dubath \& Kreykenbohm 2007). We also notice that the presentation of the imaging results in an alternative binning, for instance using a constant ratio of the high to low energies defining the band, $E_{\text {high }} / E_{\text {low }}=2$, (i.e., bands as $0.25-0.5 \mathrm{MeV}, 0.5-1.0 \mathrm{MeV}, 1.0-2.0 \mathrm{MeV}$ ) differs little, if at all, from those reported in Table 1.
}

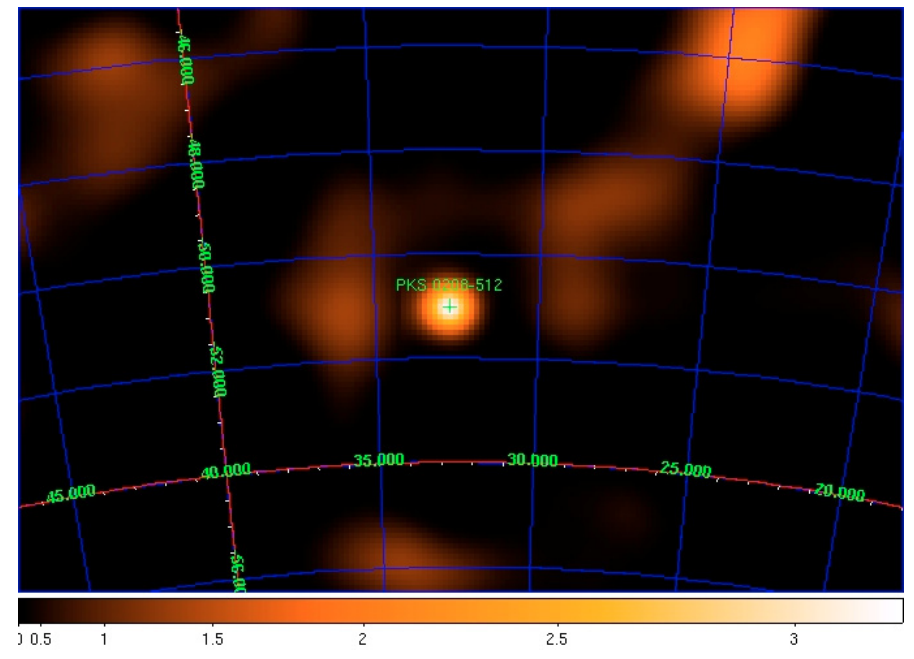

Fig. 1. SPI significance map of the sky region centered on PKS 0208512 in the $0.5-1 \mathrm{MeV}$ band is shown for an exposure of $\sim 510 \mathrm{ks}$. A source at the position of PKS 0208-512, marked by a cross, is clearly visible. The equatorial coordinates are overlaid, and the color bar at the bottom gives the detection significance scale in $\sigma$.

In order to test for a possible flare or for a temporary instrumental effect, we also analyzed the SPI data in sub-intervals. In each time interval, we found hints for an emission of the blazar, though at lower statistical significance (Table 1), indicating a likely stable $0.5-1 \mathrm{MeV}$ emission of PKS 0208-512 across the SPI observations.

For the spectral analysis we used the cataloged sky position of PKS 0208-512 and generated SPI spectra by running SPIMODFIT for narrow energy bands. The program SPIMODFIT was developed at the Max-Planck-institut für extraterrestrische Physik (MPE) for the analysis of spectra of point sources. In order to test for possible instrumental effects, we generated the spectra for different event selections, i.e., using only the SPI "singles" (events detected only in one SPI detector), 

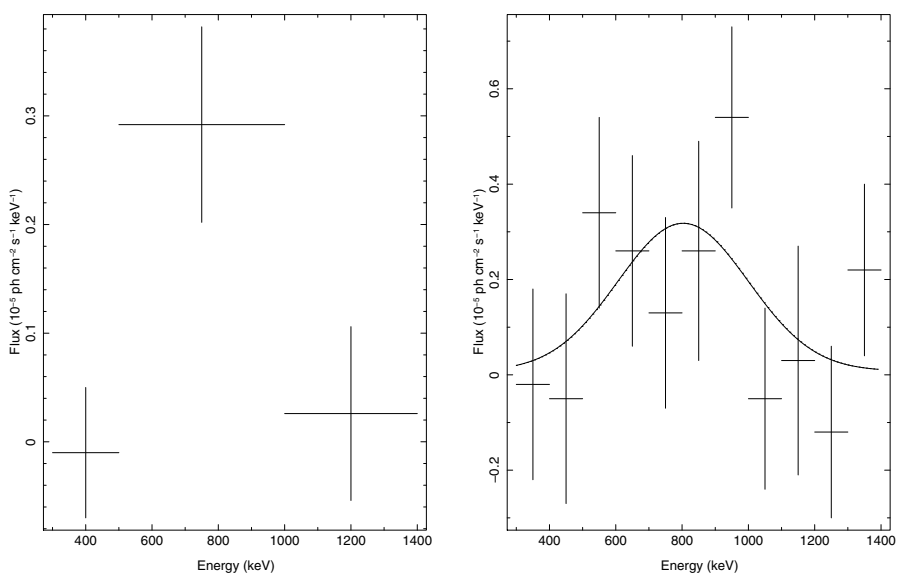

Fig. 2. Broadband INTEGRAL/SPI spectrum from the cataloged sky position of PKS 0208-512 with the energy bins of the imaging analysis (left panel) and a resolution of $100 \mathrm{keV}$ (right panel). The spectral analysis is consistent in behavior and flux with the results of the imaging analysis, showing a weak $(\sim 3.2 \sigma)$ emission between 0.5 and $1 \mathrm{MeV}$, and no evidence for the source at energies below and above. Over-plotted in the right panel is a Gaussian fit of the emission excess.

using only the SPI "multiples" (events that scattered once or twice inside SPI and so are "seen" by two or three detectors), and also using both event types combined. In the first step we generated broadband spectra by similar energy cuts as in the imaging analysis for the different event types. The spectra of the "single" and "multiple" events are statistically consistent with each other, and so independently indicate a source flux at a level of $\sim 1.5 \times 10^{-3} \mathrm{ph} \mathrm{cm}^{-2} \mathrm{~s}^{-1}$ in the 0.5 to $1 \mathrm{MeV}$ band. Both spectra are also consistent in flux with the results of the imaging analysis. Since both spectra show the same behavior, we then combined both event types for additional analyses. The resulting broadband spectrum is shown in Fig. 2 (left): it shows a flux level of $(1.46 \pm 0.45) \times 10^{-3} \mathrm{ph} \mathrm{cm}^{-2} \mathrm{~s}^{-1}$ in the $0.5-1 \mathrm{MeV}$ band, and fluxes consistent with zero at energy bands above and below the latter. We repeated this analysis with a finer spectral binning to check for a possible narrow line emission. The spectrum with a binning of $100 \mathrm{keV}$ between 0.3 and $1.4 \mathrm{MeV}$ is given in Fig. 2 (right). It shows a generally higher flux level in the 0.5 to $1.0 \mathrm{MeV}$ band, which is consistent with the imaging analysis, but shows no obvious narrow band line emission. A subsequent analysis with an even finer binning of $50 \mathrm{keV}$ resulted in the same picture. The SPI spectrum (Fig. 2) suggests a weak emission excess in the $0.5-1 \mathrm{MeV}$ band with respect to the neighboring energies. The width of this excess is constrained to $\sim 0.5 \mathrm{MeV}$ by the SPI flux measurements at energies above and below the latter band. The results of the INTEGRAL data analysis are given in Table 1.

The Swift/XRT data are well represented by a simple power law during the time period between 2005 and 2008. The spectral index remained always at $\sim 1.6$, but the flux dropped by $\sim 30 \%$ in the December 2008 observations compared to the previous ones. Table 2 gives the details on the Swift observations and on the spectral results. The combined 2008 XRT spectrum is added to the SED (Table 2, Fig. 3) to provide the contemporaneous X-ray spectrum.

\section{Discussion and summary}

In an exposure of $\sim 510 \mathrm{ks}$ SPI found hints at a $\sim 3.2-\sigma$ level, for emission from PKS 0208-512 between 0.5 and $1 \mathrm{MeV}$ at a

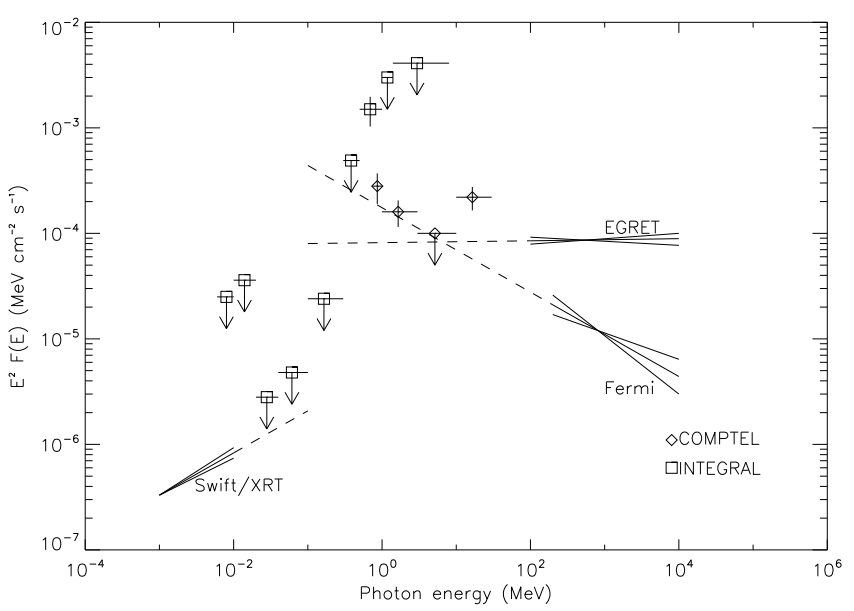

Fig. 3. Contemporaneous high-energy SED of PKS 0208-512 for two different epochs, the $C G R O$ era (COMPTEL, EGRET; 1991-2000) and recent 2008 measurements (Swift, INTEGRAL, Fermi). The COMPTEL data (open diamonds) are from Williams et al. (2001) covering the time span 1991 to 1998, and the EGRET spectral shape is from Hartman et al. (1999), measured between 1991 and 1994. The rest is from observations in 2008 by Swift/XRT (spectral shape; $0.2-10 \mathrm{keV}$ ), INTEGRAL (JEM$\mathrm{X}$, IBIS/ISGRI, SPI; open squares; $6 \mathrm{keV}-8 \mathrm{MeV}$ ), and Fermi/LAT (spectral shape; above $200 \mathrm{MeV}$ ) between November 22 and December 27. The dashed lines indicate the spectral extrapolations of the measured spectral shapes. The error bars are $1 \sigma$ and the upper limits are $2 \sigma$.

flux of $\sim 1.5 \times 10^{-3} \mathrm{ph} \mathrm{cm}^{-2} \mathrm{~s}^{-1}$, without recognizable emission at adjacent energies. A $\chi^{2}$ test on this excess gives 1.1- $\sigma$ and 2.8- $\sigma$ deviations from a linear fit for the right and the left panels of Fig. 2, respectively, consistent with the low statistics of the flux. Far from being proven, the excess remains as an interesting possibility, whose consequences warrant analysis.

To further investigate this possible emission, we analyzed the contemporaneous Fermi/LAT data (collected during the SPI revolutions 746 to 757), and generated the simultaneous energy spectrum at energies above $200 \mathrm{MeV}$. This spectrum is added to the SED (Fig. 3). The direct extrapolation of this Fermi/LAT spectrum to soft $\gamma$-rays falls short compared to the measured SPI emission in the $0.5-1 \mathrm{MeV}$ band. However, the unmeasured part of the high-energy spectra covers more than two decades, which may be misleading for a direct comparison of the two measurements. Anyway, if we take a flux of $\sim 2 \times 10^{-7} \mathrm{ph} \mathrm{cm}^{-2} \mathrm{~s}^{-1}$, as was measured contemporaneously by Fermi/LAT, and connect it to the SPI flux in the $0.5-1 \mathrm{MeV}$ band with a powerlaw shape, then the $\gamma$-ray photon index has to be as steep as 2.7. On the other hand, the SPI upper limit at $0.3-0.5 \mathrm{MeV}$ and the flux at $0.5-1 \mathrm{MeV}$ require a photon index at hard $\mathrm{X}$-ray energies harder than 0.2 . Therefore, if the measured SPI flux at $0.5-1 \mathrm{MeV}$ is canonical inverse-Compton emission, the change in photon index from hard X-rays to $\gamma$-rays has to be larger than 2.5. This is hard to account for in the current External Comptonization (EC) or Synchrotron SelfComptonization (SSC) models, unless a very unusual electron energy spectrum is assumed. Consequently this might be indicative of an additional spectral component at $0.5-1 \mathrm{MeV}$ in its SED.

A similar trend of excess emission at soft $\gamma$-rays was indicated already about ten years ago, when COMPTEL and EGRET data were combined (e.g., Blom et al. 1995; Williams et al. 2001). In Fig. 3 we include the COMPTEL spectrum 
(Williams et al. 2001), where the flux at the lowest COMPTEL energies $(0.75-1 \mathrm{MeV})$ is about a factor of 3 higher than the extrapolation of the EGRET spectrum. The results from COMPTEL/EGRET and INTEGRAL/SPI/Fermi are independently derived and may mutually strengthen each other.

The broad emission feature between 0.5 and $1 \mathrm{MeV}$, if emitted by the blazar, could be understood as a broadened and Doppler blue-shifted pair annihilation radiation, emitted by a jet containing an electron-positron pair plasma. Assuming such a scenario, we derive by the arguments below the following estimates on the blazar jet of PKS 0208-512 and compare these to measured parameters using data from other wavelengths.

The central energy $E$ of this emission feature is related to the kinematics of the jet, $E=\gamma_{\min } D /(1+z) \times 511 \mathrm{keV}$, where $\gamma_{\min }$ is the minimum Lorentz factor of the electrons and positrons in the jet, and $D$ is the Doppler boosting factor (Böttcher \& Schlickeiser 1996). To estimate $E$, we fitted the right panel of Fig. 2 with a Gaussian shape. The central energy was derived as $803_{-291}^{+233} \mathrm{keV}$, well within the energy range of $0.5-1.0 \mathrm{MeV}$, and the width was $\sim 200 \mathrm{keV}$ without a well-constrained error. The reduced $\chi^{2}$ for the fit was 1.1 for 7 degrees of freedom. The apparent speed of the relativistic bulk motion of PKS 0208512 was measured with VLBI as $\sim(2.4 \pm 3.1) h^{-1}$ c (Tingay et al. 2002). By taking $\gamma_{\min }=1, h=1$ and $D \sim 3$, the bulk Lorentz factor $\Gamma$ could then be estimated to $\sim 2.6_{-0.9}^{+4.1}$. Subsequently, the offset angle $\theta$ between our line of sight and the jet became less than $\sim 19^{\circ}$. We note that a caveat on the estimation of this offset angle is that the VLBI measurement of the bulk motion was not contemporary.

The density of a relativistic electron-positron pair plasma can be up to $\sim 10^{10}\left(L_{\mathrm{e}^{ \pm}} D^{-4} / V_{\mathrm{b}}\right)^{1 / 2} \mathrm{~cm}^{-3}$ (Roland \& Hermsen 1995). Here the beam was assumed to consist of $\mathrm{e}^{ \pm}$and the annihilation emission could dominate in a volume $V_{\mathrm{b}}$, which was inferred from the time variability in $\gamma$-rays. The relativistic $\mathrm{e}^{ \pm}$in the beam were supposed to have a power law distribution with an index $\sim 3$ (Roland \& Hermsen 1995). The annihilation luminosity could then be estimated under an approximation of the annihilation rate (Coppi \& Blandford 1990). If we take $L_{\mathrm{e}^{ \pm}}$as the measured luminosity at the $0.5-1 \mathrm{MeV}$ band, $D=3$, and a lower limit of $V_{\mathrm{b}}=\pi R_{\mathrm{b}}^{2} L_{\mathrm{b}} \sim 5 \times 10^{46} \mathrm{~cm}^{3}$, we derive an upper limit of $\sim 10^{9} \mathrm{~cm}^{-3}$ for the density of electron-positron population in the jet. Here we took the radius of the beam as $R_{\mathrm{b}} \sim 20 \mathrm{GM} / \mathrm{c}^{2}$ (Marcowith et al. 1995), the beam length $L_{\mathrm{b}}$ of $\sim 100$ light days due to the variability time scale of the $\gamma$ rays flare observed by Fermi in 2008, and the central black hole mass as $M \sim 10^{8} M_{\odot}$, which is typical for a blazar. The $L_{\mathrm{e}^{ \pm}}$ is about $6.3 \times 10^{48} \mathrm{erg} / \mathrm{s}$, given a redshift $z=1.003$ (we used $\left.H_{0}=75 \mathrm{~km} \mathrm{~s}^{-1} \mathrm{Mpc}^{-1}, q_{0}=0.5\right)$. By taking the Eddington limit of $1.3 \times 10^{38} M / M_{\odot}$ erg/s, the central black hole mass was estimated to be larger than $6 \times 10^{8} M_{\odot}$. Using this mass, the upper limit for the density of the electron-positron population in the jet decreases to $\sim 10^{8} \mathrm{~cm}^{-3}$. A significant number of cold leptons, responsible for the annihilation line, may generate the bulk spectral feature in the soft X-ray energy range via Comptonization off the surrounding UV photons, as discussed in e.g. Sikora et al. (1997), where the emission peak is estimated at $\sim(\Gamma / 10)^{2} \mathrm{keV}$. By taking $\Gamma \sim 2.6$, the emission peak is about $0.07 \mathrm{keV}$, well below the Swift/XRT energy domain. We notice that the XRT spectrum can be well fitted by a simple power-law shape, without any further components.

The $\gamma$-ray flux above $100 \mathrm{MeV}$, averaged from August to October 2008, was a factor of $\sim 3.5$ lower than roughly ten years (1991-2000) ago during the CGRO era (Hartman et al. 1999; Abdo et al. 2009). The Swift/XRT observations show that the
X-ray flux (2-10 keV) of PKS 0208-512 also decreased by more than $50 \%$ in comparison to the BeppoSAX observation in 2001 (Tavecchio et al. 2002; Donato et al. 2005). However, if the soft $\gamma$-ray excess observed by SPI at $0.5-1 \mathrm{MeV}$ is emitted by the blazar, this component is even brighter now than during the CGRO times. A possible explanation could be that the $\gamma$-rays generated by inverse-Compton emission of the nonthermal pairs are more dependent on the Doppler factor than the thermal annihilation radiation in the jet (Skibo et al. 1997). Therefore, blazars, viewed at a moderate jet offset-angle, can show a significant blue-shifted annihilation radiation, which outshines the continuum emission (Skibo et al. 1997). This may actually have happened in PKS 0208-512: a viewing angle of less than $\sim 19^{\circ}$ matches the prediction for this viewing-angle scenario. The moderate distance at a redshift $z \sim 1$ makes PKS 0208-512 detectable in $\gamma$-rays, despite its relatively large jet offset-angle.

Further monitoring of this source by INTEGRAL, Fermi/LAT and Swift, as well as by the planned NeXT high energy astronomy mission with a more sensitive Compton telescope below $600 \mathrm{keV}$ and a broad band capability down to about $0.3 \mathrm{keV}$ should clarify this picture. If confirmed, the monitoring will be able to constrain the pair density in the PKS 0208-512 jet directly from observing the annihilation radiation of the pair plasma.

Acknowledgements. We are grateful to the anonymous referee for his/her comments which were of great helpful to polish the paper. This work was subsidized by the National Natural Science Foundation of China, and the CAS key Project KJCX2-YW-T03 and the 973 Program 2009CB824800. D.F.T. has been supported by grants AYA2009-07391 and SGR2009- 811. J.-M. Wang and S.-N. Zhang thank the Natural Science Foundation of China for support via NSFC$10325313,10733010,10725313$ and 10821061. Shu Zhang would like to thank INTEGRAL and Swift for approving INTEGRAL proposal No. 0620052 and two SWFIT ToO (Target of Opportunity) proposals (ID No. 35002), and for subsequently carrying out the observations of roughly $700 \mathrm{ks}$ to support this research.

\section{References}

Abdo, A. A., Ackermann, M., Ajello, M., et al. 2009, ApJ, 700, 597 Acciari, V. A., Aliu, E., Arlen, T., et al. 2009, Science, 325, 444

Aharonian, F., Akhperjanian, A. G., Bazer-Bachi, A. R., et al. 2006, Science, 314,1424

Aharonian, F., Akhperjanian, A. G., Bazer-Bachi, A. R., et al. 2007, ApJ, 664, L71

Albert, J., Aliu, E., Anderhub, H., et al. 2007, ApJ, 669, 862

Atwood, W. B., Abdo, A. A., Ackermann, M., et al. 2009, ApJ, 697, 1071

Barthelmy, S. D., Barbier, L. M., Cummings, J. R., et al. 2005, Space Sci. Rev., 120,143

Begelman, M. C., Blandford, R. D., \& Rees, M. J. 1984, Rev. Mod. Phys., 56, 255

Bertsch, D. L., Dingus, B. L., Fichtel, C. E., et al. 1993, ApJ, 405, L21

Bloemen, H., Bennett, K., Blom, J. J., et al. 1995, A\&A, 293, L1

Blom, J. J., Bennett, K., Bloemen, H., et al. 1995, A\&A, 298, L33

Böttcher, M., \& Schlickeiser, R. 1996, A\&A, 306, 86

Bouchet, L., Roques, J.-P., Mandrou, P., et al. 2005, ApJ, 635, 1103

Bouchet, L., Jourdain, E., Roques, J.-P., et al. 2008, ApJ, 679, 1315

Burrows, D. N., David, N., Hill, J. E., et al. 2005, Space Sci. Rev., 120, 165

Buxton, M., Bailyn, C., Coppi, P., et al. 2008, ATel, 1751

Celotti, A., \& Ghisellini, G. 2008, MNRAS, 385, 283

Coppi, P. S., \& Blandford, R. D. 1990, MNRAS, 245, 453

Donato, D., Sambruna, R. M., \& Gliozzi, M. 2005, A\&A, 433, 1163

Dubath, P., \& Kreykenbohm, I. 2007, SPI Analysis User Manual

Dunn, R. J. H., Fabian, A. C., \& Celotti, A. 2006, MNRAS, 372, 1741

Gehrels, H., Chincarini, G., Giommi, P., et al. 2004, ApJ, 611, 1005

Ghisellini, G., Tavecchio, F., Foschini, L., et al. 2010, MNRAS, 402, 497

Hartman, R. C., Bertsch, D. L., Bloom, S. D., et al. 1999, ApJS, 123, 79

Hirotani, K., Iguchi, S., Kimura, M., \& Wajima, K. 2000, ApJ, 545, 100 
A\&A 514, A69 (2010)

Kino, M., \& Takahara, F. 2004, MNRAS, 349, 336

Kn̈odlseder, J., Weidenspointner, G., Jean, P., et al. 2007, ESA SP-622, 13 [arXiv:0712.1668]

Lebrun, F., Leray, J. P., Lavocat, P., et al. 2003, A\&A, 411, L141

Lobanov, A. P., \& Zensus, J. A. 2001, Science, 294, 128

Lund, N., Budtz-Jørgensen, C., Westergaard, N. J., et al. 2003, A\&A, 411, L231

Marcowith, G., Henri, G., \& Pelletier, G. 1995, MNRAS, 277, 681

Marscher, A. P., Jorstad, S. G., Gómez, J. L., et al. 2007, ApJ, 665, 232

Mas-Hesse, J. M., Giménez, A., Culhane, J. L., et al. 2003, A\&A, 411, L261

von Montigny, C., Bertsch, D. L., Chiang, J., et al. 1995, ApJ, 440, 525

Petry, D., Beckmann, V., Halloin, H., \& Strong, A. 2009, A\&A, 507, 549

Reynolds, C. S., Fabian, A. C., Celotti, A., \& Rees, M. J. 1996, MNRAS, 283, 873

Roland, J., \& Hermsen, W. 1995, A\&A, 297, L9

Roming, P. W. A., Kennedy, T. E., Mason, K. O., et al. 2005, Space Sci. Rev., 120,95
Schwartz, D. A., Marshall, H. L., Lovell, J. E. J., et al. 2006, ApJ, 640, 592 Schwartz, D. A. 2007, Rev. Mex. Astron. Astrofis., 27, 102

Sikora, M., Madejski, G., Moderski, R., \& Poutanen, J. 1997, ApJ, 484, 108 Skibo, J. G., Dermer, C. D., \& Schlickeiser, R. 1997, ApJ, 483, 56

Stacy, J. G., Vestrand, W. T., \& Sreekumar, P. 2003, ApJ, 598, 216

Tavecchio, F., Maraschi, L., Ghisellini, G., et al. 2002, ApJ, 575, 137

Tingay, S. J., Reynolds, J. E., Tzioumis, A. K., et al. 2002, ApJS, 141, 311 Tosti, G. 2008, ATel, 1759

Ubertini, P., Lebrun, F., Di Cocco, G., et al. 2003, A\&A, 411, L131

Vedrenne, G., Roques, J.-P., Schönfelder, V., et al. 2003, A\&A, 411, L63

Wardle, J. F. C., Homan, D. C., Ojha, R., \& Roberts, D. H. 1998, Nature, 395, 457

Williams, O. R., Bennett, K., Collmar, W., et al. 2001, in Exploring the gammaray universe, Proceedings of the Fourth INTEGRAL Workshop, ESA SP-459, 357 